\title{
Can Smartphone Sensors Enhance Kinect Experience?
}

\author{
[Extended Abstract]
}

\author{
Rufeng Meng, Jason Isenhower, Chuan Qin, Srihari Nelakuditi \\ Computer Science and Engineering, University of South Carolina, Columbia, SC 29208 \\ \{mengr, isenhowj, qinc, srihari\}@cse.sc.edu
}

\begin{abstract}
Kinect has become quite popular for gaming as it tracks players' natural gestures without a controller like other gaming systems. But it has some inherent limitations such as occlusion problem and fails to track a player accurately if there is an obstacle. In this work, we propose to leverage smartphone to supplement Kinect. We explore how to fuse information from Kinect's tracking with the smartphone's sensor readings to improve Kinect gaming experience.
\end{abstract}

\section{Categories and Subject Descriptors}

I.4.8 [Image Processing and Computer Vision]: Scene Analysis; K.8.0 [Personal Computing]: General

\section{Keywords}

Kinect, Smartphone, Games, Sensor Fusion

\section{INTRODUCTION AND MOTIVATION}

Kinect is one of the most successful systems that promote the use of natural and intuitive gestures from the users. Kinect employs cameras and depth sensor (see Fig. 1) to track the user's body in real time. It uses an infrared sensor to project light patterns on users in front of it. The light pattern is distorted by the users due to the different distance from the users to Kinect. The depth sensor uses the distorted light pattern to calculate the depth (i.e. the distance from Kinect to user) based on some reference frame corresponding to the projected light pattern [1].

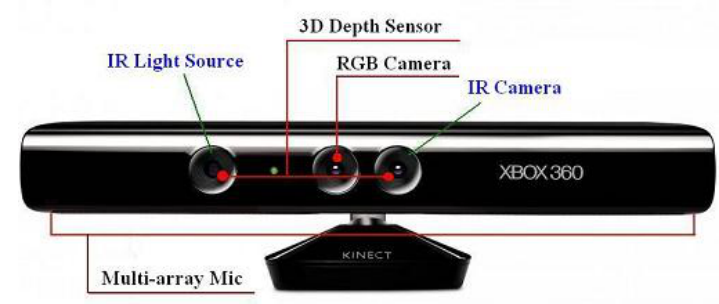

Figure 1: Kinect Sensors

Although Kinect is very intuitive, it has some inherent limitations. One of the major problems Kinect suffers from

Copyright is held by the author/owner(s).

MobiHoc'12, June 11-14, 2012, Hilton Head Island, SC, USA.

ACM 978-1-4503-1281-3/12/06. is occlusion. When some joints of the body are hidden by any obstacle, Kinect loses its tracking and tries to infer the coordinates of the hidden joints. But in many instances, its inference can be quite inaccurate. Kinect also has a relatively small view range (horizontal view: 57 degrees, vertical view: 43 degrees) which limits its ability to simultaneously track multiple users (currently Kinect can only actively track 2 users and detect 4 additional users at the same time). Kinect is designed for relatively short distance usage (less than 5 meters), which limits its applicability in wider spaces. Although Kinect has other limitations and drawbacks, we plan to focus on these three aspects.

We propose to enhance the capabilities of Kinect by leveraging smartphones. Nowadays, many people have smartphones and it is reasonable to expect that they carry them (in pocket or on belt) even when they are playing games. Almost all the smartphones have accelerometer, gyroscope, microphone, etc. When the player is moving, the smartphone sensors can produce information related to the movement. For example, when the player is kicking or rotating, the accelerometer and gyroscope can potentially measure the real-time acceleration and rotation speed of the player's action. Although Kinect can track the player's movement, it may not know how hard a player is doing a kicking action or the real-time speed of a rotation of the body. The smartphone can help to improve the accuracy and sensitivity of Kinect and potentially mitigate its limitations.

We plan to fuse the data from Kinect and smartphone's sensors to enhance the capability and user experience of Kinect system for both the developers and players.

\section{RELATED WORK}

Some work in combining Kinect with smartphones has been done in literature. The authors of [2] built an interactive augmented reality system, which uses Kinect to capture the motion, depth map and real images. They also use smartphone, which is attached to a cap, to measure the heading, pitch and roll of the head from the phone's accelerometer and compass. [3] presents a solution which utilizes a Kinect to sense gesture input and uses Android smartphones to interact with the display wall. In the system shown in [4], Kinect is used to track the user behavior, and the acceleration information from mobile phone is utilized to complement the coarse contextual data of Kinect.

\section{OUR APPROACH}

Kinect continuously tracks and records the video image, skeletal joint coordinates, and depth information for the 
users in its viewing range. This information is transmitted to a Xbox in real-time. Fig. 2 illustrates the proposed smartphone-enhanced Kinect system. The user carries smartphone in pocket when (s)he plays games or uses Kinect applications. In our design, the smartphone also connects with the Xbox. The sensor readings from the smartphone are also sent to the Xbox in real-time. The Xbox fuses the data from Kinect and smartphone sensors to better track the users.

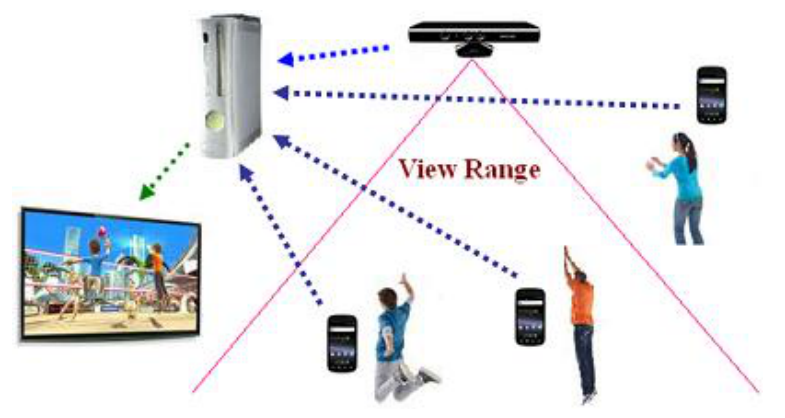

Figure 2: Smartphone-enhanced Kinect System

We propose to enhance Kinect system in three aspects with the aid of smartphones: broaden its tracking range, improve its accuracy, increase its hearing distance.

Current Kinect systems can only actively track 2 users and detect 4 additional users simultaneously. When a user goes out of its view, Kinect will stop tracking the user and the character corresponding to the user in the game might be destroyed. With the aid of a smartphone, even when the user runs out of Kinect's view, Xbox can still detect that the user is still playing by analyzing the pattern of the smartphone's sensor readings. Moreover, since Kinect has tracked this user in the past, Xbox can remember some characteristics (e.g. height, shoulder width) of the user. This allows Xbox to construct or maintain the character in the game corresponding to the user who is outside Kinect's view. Smartphone's sensors could tell whether the user is spinning, jumping, etc., enabling the Xbox to render the character's movements. In this way, the user could still be in the game even if she has moved out from Kinect's view temporarily. In other words, smartphone broadens Kinect's tracking range which makes it possible to detect and track more users simultaneously.

Kinect suffers from occlusion problem. When some joints of the user are hidden, Kinect loses its tracking and tries to infer the coordinates for these hidden joints. But in many instances, this inference is not accurate, sometimes it is totally incorrect. We do a simple experiment to study this problem. Figure 3 shows the user doing a spin in front of the Kinect. A smartphone is in the user's pocket and the built-in gyroscope is recording the user's rotation. The two graphs in the bottom of Figure 3 show the rotation result calculated from Kinect's skeletal joint coordinates (we use the two joints on left and right shoulders to calculate the rotation of the user) and smartphone's gyroscope readings. From the graph, it is evident that there are some moments when the user's joints are unseen, Kinect fails to track and gives inaccurate joint coordinates, which leads to the rugged circle. On the other hand, smartphone's gyroscope can record the user's rotation in any direction and measure the rotation accurately, which leads to the perfect circle at the bottom right of Figure 3.

Each smartphone has an embedded microphone. When

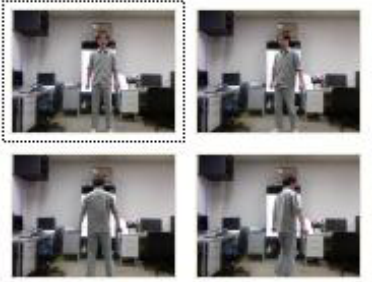

(a)
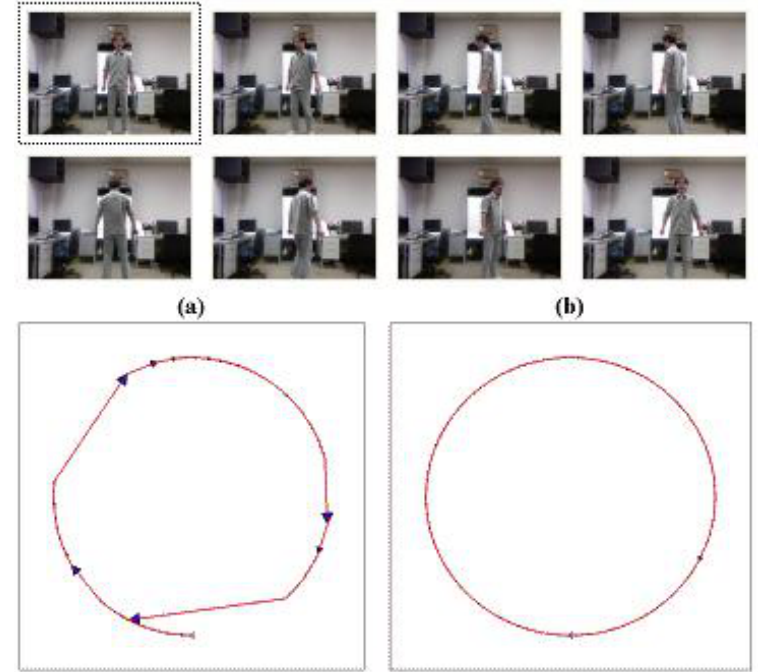

(b)

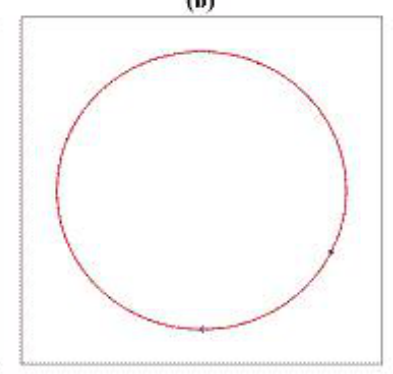

Figure 3: User spins (shown in the top). Related rotation retrieved from Kinect (bottom left) and smartphone's gyroscope (bottom right).

the smartphone is carried by the user, its microphone is much closer to the user compared to Kinect's microphones. So the smartphone could collect the voice sample and transmit to Xbox to fuse with the voice recorded by Kinect. In this way, we can improve Kinect's hearing distance.

\section{ONGOING WORK}

We are currently analyzing and fusing the sensor data from both Kinect and smartphone. We plan to develop a prototype and do more experiments to see in which aspects and to what extent the smartphone sensors can help enhance the capabilities of Kinect. Depending on how a user carries the smartphone, it may only measure the movement of one particular part of the body (one joint in Kinect's viewpoint). Different games and applications require the movement of different parts of the user's body. Therefore, we need to investigate which categories of games and applications are likely to benefit most from smartphone-enhanced Kinect system. Our long-term goal is to create a set of enhanced Kinect API based on current Kinect SDK for developers, which allows subtler and smoother control over the characters in games as well as actions in applications.

\section{REFERENCES}

[1] John MacCormick, "How does the kinect work?," http://users.dickinson.edu/ jmac/ selected-talks/kinect.pdf.

[2] L. Vera, J. Gimeno, I. Coma, and M. Fernández, "Augmented mirror: interactive augmented reality system based on kinect," Human-Computer Interaction-INTERACT 2011, pp. 483-486, 2011.

[3] V. Kulkarni, H. Wu, and Y. Jin, "Bayscope: Evaluating interaction techniques for large wall-sized displays," in CHI 2012, Austin, TX, USA, May 2012, ACM.

[4] L. Norrie and R. Murray-Smith, "Virtual sensors: rapid prototyping of ubiquitous interaction with a mobile phone and a kinect," in ACM Mobile HCI, 2011. 